\title{
Application of Sumudu Decomposition Method to Solve Nonlinear System Volterra Integrodifferential Equations
}

\author{
Hassan Eltayeb, ${ }^{1}$ Adem Kılıçman, ${ }^{2}$ and Said Mesloub ${ }^{1}$ \\ ${ }^{1}$ Mathematics Department, College of Science, King Saud University, P.O. Box 2455, Riyadh 11451, Saudi Arabia \\ ${ }^{2}$ Department of Mathematics, Institute for Mathematical Research, Universiti Putra Malaysia, (UPM), \\ 43400 Serdang, Selangor, Malaysia
}

Correspondence should be addressed to Said Mesloub; mesloubs@yahoo.com

Received 18 February 2014; Accepted 4 April 2014; Published 28 April 2014

Academic Editor: Mohamed Boussairi Jleli

Copyright (c) 2014 Hassan Eltayeb et al. This is an open access article distributed under the Creative Commons Attribution License, which permits unrestricted use, distribution, and reproduction in any medium, provided the original work is properly cited.

\begin{abstract}
We develop a method to obtain approximate solutions for nonlinear systems of Volterra integrodifferential equations with the help of Sumudu decomposition method (SDM). The technique is based on the application of Sumudu transform to nonlinear coupled Volterra integrodifferential equations. The nonlinear term can easily be handled with the help of Adomian polynomials. We illustrate this technique with the help of three examples and results of the present technique have close agreement with approximate solutions which were obtained with the help of Adomian decomposition method (ADM).
\end{abstract}

\section{Introduction}

The linear and nonlinear Volterra integral equations arise in many scientific fields such as the population dynamics, spread of epidemics, and semiconductor devices; for more details, see [1]. The scientists in different branches of science have been trying to solve this kind of problems; however, finding an exact solution is not easy due to the nonlinear part of these type groups of equations. Different analytical methods have been developed and applied to find a solution. For example, Adomian has introduced a so-called decomposition method for solving algebraic, differential, integrodifferential, differential-delay, and partial differential equations. In the nonlinear case for ordinary differential equations and partial differential equations, the method has the advantage of dealing directly with the problem $[2,3]$. These equations are solved without transforming them to equivalent form which is more simple. The method avoids linearization, perturbation, discretization, or any unrealistic assumptions; see $[4,5]$. It was also suggested in $[6]$ that the noise terms appear always for inhomogeneous equations. Thus, most recently, Wazwaz [7] established a necessary condition that is essentially needed to ensure the appearance of "noise terms" in the inhomogeneous equations.

The integral transform has been used to solve many different types of differential and integrodifferential equations. For similar problems, Sumudu transform was introduced and further applied to several ODEs as well as PDEs. For example, in [8], this transform was applied to the one-dimensional neutron transport equation. In [9], Sumudu transform was extended to the distributions and some of their properties were also studied in [10]. Recently, Kılıçman et al. applied this transform to solve the system of differential equations (see [11]), since there are some interesting properties that Sumudu transform satisfies such as if

$$
f(t)=\sum_{n=0}^{\infty} a_{n} t^{n}, \quad \text { then } F(u)=\sum_{n=0}^{\infty} n ! a_{n} u^{n}
$$

see $[12]$.

In the present paper, the intimate connection between Sumudu transform theory and decomposition method arising in the solution of nonlinear Volterra integrodifferential equation is demonstrated. 
During the study, we use Sumudu transform which is defined over the set of the following functions:

$$
\begin{aligned}
A=\left\{f(t): \exists M, \tau_{1}, \tau_{2}>\right. & 0,|f(t)|<M e^{t / \tau_{j}}, \\
& \text { if } \left.t \in(-1)^{j} \times[0, \infty)\right\}
\end{aligned}
$$

by the following formula:

$$
\begin{aligned}
G(u) & =S[f(t) ; u] \\
& :=\int_{0}^{\infty} f(u t) e^{-t} d t, \quad u \in\left(-\tau_{1}, \tau_{2}\right) .
\end{aligned}
$$

Theorem 1. Let $f(t)$ be in $A$, and let $G^{n}(u)$ denote Sumudu transform of $n$th derivative, $f^{n}(t)$ of $f(t)$; then, for $n \geq 1$,

$$
G^{n}(u)=\frac{G(u)}{u^{n}}-\sum_{k=0}^{n-1} \frac{f^{(k)}(0)}{u^{n-k}} .
$$

For more details, see [13].

Recall that Sumudu transform of the convolution product $(f * g)(x)$ is given by

$$
\begin{aligned}
S & {[(f * g)(x ; u)] } \\
& =S\left[\int_{0}^{x} f(x-t) g(t) d t\right]=u F(u) G(u) .
\end{aligned}
$$

We consider general nonlinear Volterra integrodifferential equation:

$$
\frac{d^{n} U}{d x^{n}}=f(x)+\int_{0}^{x} K(x-t) F(U(t)) d t .
$$

To solve the nonlinear Volterra integrodifferential equations by using Sumudu transform method, it is essential to use Sumudu transforms of the derivatives of $U(x)$. We can easily show that

$$
\begin{aligned}
S\left[\frac{d^{n} U}{d x^{n}}\right]= & \frac{1}{u^{n}} S[U(x)] \\
& -\frac{1}{u^{n}} U(0)-\frac{1}{u^{n-1}} U^{\prime}(0)-\cdots-\frac{U^{n-1}(0)}{u} .
\end{aligned}
$$

Applying Sumudu transform to both sides of (7) gives

$$
\begin{aligned}
\frac{1}{u^{n}} S[U(x)]-\frac{1}{u^{n}} U(0) & \\
& -\frac{1}{u^{n-1}} U^{\prime}(0)-\cdots-\frac{U^{(n-1)}(0)}{u} \\
= & S[f(x)]+u S(K(x-t)) S(F(u(t))) ;
\end{aligned}
$$

by arrangement, we have

$$
\begin{aligned}
S[U(x)]= & u^{n} S[f(x)]+U(0) \\
& +u U^{\prime}(0)+\cdots+u^{n-1} U^{(n-1)}(0) \\
& +u^{n+1} S(K(x-t)) S(F(u(t))) .
\end{aligned}
$$

The second step in Sumudu decomposition method is that we represent the solution as an infinite series given by

$$
U(x, \lambda)=\sum_{i=0}^{\infty} U_{i}(x)
$$

and the nonlinear term can be decomposed as

$$
F(u(t))=\sum_{i=0}^{\infty} A_{i}
$$

where $A_{i}$ are Adomian polynomials [7] of $U_{0}, U_{1}, U_{2}, \ldots, U_{n}$ and they can be calculated by the following formula:

$$
A_{i}=\frac{1}{i !} \frac{d^{i}}{d \lambda^{i}}\left[F\left(\sum_{i=0}^{\infty} \lambda^{i} u_{i}\right)\right]_{\lambda=0}, \quad i=0,1,2, \ldots
$$

where the so-called Adomian polynomials $A_{n}$ can be evaluated for all forms of nonlinearity. General formula (12) can be easily used as follows.

Assuming that the nonlinear function is $F(u(x))$, therefore, by using (12), Adomian polynomials are given by

$$
\begin{aligned}
A_{0} & =F\left(U_{0}\right), \quad A_{1}=U_{1} F^{\prime}\left(U_{0}\right) \\
A_{2} & =U_{2} F^{\prime}\left(U_{0}\right)+\frac{1}{2 !} U_{1}^{2} F^{\prime \prime}\left(U_{0}\right) \\
A_{3}= & U_{3} F^{\prime}\left(U_{0}\right)+U_{1} U_{2} F^{\prime \prime}\left(U_{0}\right)+\frac{1}{3 !} U_{1}^{3} F^{\prime \prime \prime}\left(U_{0}\right) \\
A_{4}= & U_{4} F^{\prime}\left(U_{0}\right)+\left(\frac{1}{2 !} U_{2}^{2}+U_{1} U_{3}\right) F^{\prime \prime}\left(U_{0}\right) \\
& \quad+\frac{1}{2 !} U_{1}^{2} U_{2} F^{\prime \prime \prime}\left(U_{0}\right)+\frac{1}{4 !} U_{1}^{4} U_{2} F^{(i v)}\left(U_{0}\right) .
\end{aligned}
$$

Substitution of (10) and (11) into (9) yields

$$
\begin{aligned}
S\left[\sum_{i=0}^{\infty} U_{i}(x)\right] \\
=u^{n} S[f(x)]+U(0)+u U^{\prime}(0) \\
\quad+\cdots+u^{n-1} U^{(n-1)}(0) \\
\quad+u^{n+1} S(K(x-t)) S\left(\left[\sum_{i=0}^{\infty} A_{i}\right]\right) .
\end{aligned}
$$

On comparing both sides of (14) and by using standard ADM we have

$$
\begin{aligned}
& S\left[U_{0}(x)\right] \\
& =u^{n} S[f(x)]+U(0) \\
& \quad+u U^{\prime}(0)+\cdots+u^{n-1} U^{(n-1)}(0) .
\end{aligned}
$$

Then it follows that

$$
\begin{aligned}
S\left[U_{1}(x)\right] & =u^{n+1} S(K(x-t)) S\left(A_{0}(x)\right), \\
S\left[U_{2}(x, t)\right] & =u^{n+1} S(K(x-t)) S\left(A_{1}(x)\right) .
\end{aligned}
$$


More generally way, we have

$$
S\left[U_{i+1}(x)\right]=u^{n+1} S(K(x-t)) S\left(A_{i}(x)\right) \quad i \geq 0 .
$$

Combined Sumudu transform-Adomian decomposition method for solving nonlinear Volterra integrodifferential equations of the second kind will be illustrated by studying the following example.

Example 2. Consider solving the nonlinear Volterra integrodifferential equation by using combined Sumudu transform-Adomian decomposition method:

$$
\begin{aligned}
U^{\prime \prime}(x)= & -1-\frac{1}{3}(\sin (x)+\sin (2 x)) \\
& +\cos (x)+\int_{0}^{x} \sin (x-t) U^{2}(t) d t, \\
U^{\prime}(0)= & -1, \quad U(0)=1 .
\end{aligned}
$$

By applying Sumudu transform to both sides of (18) we obtain

$$
\begin{aligned}
\frac{1}{u^{2}} S[U(x)]-\frac{1}{u^{2}} U(0)-\frac{1}{u} U^{\prime}(0) \\
=-1-\frac{1}{3}\left(\frac{u}{1+u^{2}}+\frac{2 u}{1+4 u^{2}}+\frac{1}{1+u^{2}}\right) \\
\quad+\frac{u^{2}}{1+u^{2}} S\left[U^{2}(t)\right]
\end{aligned}
$$

or equivalently

$$
\begin{aligned}
S[U(x)]= & 1-u-u^{2} \\
& -\frac{1}{3}\left(\frac{u^{3}}{1+u^{2}}+\frac{2 u^{3}}{1+4 u^{2}}+\frac{u^{2}}{1+u^{2}}\right) \\
& +\frac{u^{4}}{1+u^{2}} S\left[U^{2}(t)\right] .
\end{aligned}
$$

Substituting the series assumption for $U(s)$ and Adomian polynomials for $u^{2}(x)$ as given above in (10) and (12), respectively, by using the recursive relation equation (14), we obtain

$$
\begin{aligned}
& S\left[U_{0}(x)\right] \\
& \quad=1-u-u^{2}-\frac{1}{3}\left(\frac{u^{3}}{1+u^{2}}+\frac{2 u^{3}}{1+4 u^{2}}+\frac{u^{2}}{1+u^{2}}\right) .
\end{aligned}
$$

Recall that Adomian polynomials for $F(u(x))=u^{2}(x)$ are given by

$$
\begin{aligned}
& A_{0}=U_{0}^{2}, \quad A_{1}=2 U_{0} U_{1}, \\
& A_{2}=2 U_{0} U_{2}+U_{1}^{2}, \quad A_{3}=2 U_{0} U_{3}+2 U_{1} U_{2} .
\end{aligned}
$$

Taking the inverse Sumudu transform of both sides of the first part of (21) and using the recursive relation equation (21) give

$$
\begin{aligned}
U_{0}(x)= & -1+x+\frac{1}{2} x^{2}-\frac{1}{6} x^{3}-\frac{1}{12} x^{4} \\
& +\frac{1}{40} x^{5}+\frac{1}{360} x^{6}-\frac{11}{5040} x^{7}+\cdots, \\
U_{1}(x)= & \frac{1}{24} x^{4}-\frac{1}{60} x^{5}-\frac{1}{720} x^{6}+\frac{1}{504} x^{7}+\cdots .
\end{aligned}
$$

By using (10), we obtain the series solution as follows:

$$
\begin{aligned}
U(x)= & \left(x-\frac{1}{3 !} x^{3}+\frac{1}{5 !} x^{5}-\frac{1}{7 !} x^{7}+\cdots\right) \\
& -\left(1-\frac{1}{2 !} x^{2}+\frac{1}{4 !} x^{4}-\frac{1}{6 !} x^{6}+\cdots\right)
\end{aligned}
$$

The exact solution is given by

$$
U(x)=\sin (x)-\cos (x) .
$$

In the next problem, we apply the combined Sumudu transform-Adomian decomposition method. The standard form of the nonlinear Volterra integrodifferential equation of the first kind is given by

$$
\begin{aligned}
& \int_{0}^{x} k_{1}(x-t) F(U(t)) d t \\
& \quad+\int_{0}^{x} k_{2}(x-t) U^{n}(t) d t=f(x) .
\end{aligned}
$$

On using Sumudu transform for both sides of (26) and using (5), we get

$$
S\left(k_{1}(x) * F(U(x))\right)+S\left(k_{2}(x) * U^{(n)}(x)\right)=S(f(x)) .
$$

So we have

$$
\begin{aligned}
& u K_{1}(u) S(F(U(x)))+u K_{2}(u) S\left(U^{(n)}(x)\right)=F(u) \\
& S[U(x)] \\
& \quad=u^{n-1}\left(\frac{F(u)+K_{2}(u) \Psi(u)-u K_{1}(u) S(F(U(x)))}{K_{2}(u)}\right),
\end{aligned}
$$

where

$$
\Psi(u)=\frac{1}{u^{n-1}} U(0)+\frac{1}{u^{n-2}} U^{\prime}(0)+\cdots+U^{(n-1)}(0) .
$$

We now use Adomian decomposition method to handle (29). Substituting (10) and (11) into (29),

$$
\begin{aligned}
S\left[\sum_{i=0}^{\infty} U_{i}(x)\right] \\
=\frac{u^{n-1} S[f(x)]}{K_{2}(u)}+U(0)+u U^{\prime}(0) \\
\quad+\cdots+u^{n-1} U^{(n-1)}(0)-u^{n} \frac{K_{1}(u)}{K_{2}(u)} S\left(\left[\sum_{i=0}^{\infty} A_{i}\right]\right) .
\end{aligned}
$$


The Adomian decomposition method admits the use of the following recursive relation:

$$
\begin{aligned}
U_{0}(x)= & \frac{u^{n-1} S[f(x)]}{K_{2}(u)}+U(0)+u U^{\prime}(0) \\
& +\cdots+u^{n-1} U^{(n-1)}(0), \\
U_{k+1}(x)= & -u^{n} \frac{K_{1}(u)}{K_{2}(u)} S\left(A_{k}\right), \quad k \geq 0 .
\end{aligned}
$$

Example 3. Solve the following nonlinear Volterra integrodifferential equation of the first kind by the combined Sumudu transform-Adomian decomposition method:

$$
\begin{aligned}
\int_{0}^{x} & (x-t) U^{2}(t) d t+\int_{0}^{x} e^{(x-t)} U^{\prime}(t) d t \\
& =-\frac{1}{4}-\frac{1}{2} x+x e^{x}+\frac{1}{4} e^{2 x}, \quad U(0)=1
\end{aligned}
$$
have

By applying Sumudu transforms to both sides of (33), we

$$
\begin{aligned}
S(U(x))= & 1-\frac{(1-u)}{4}-\frac{1}{2} u(1-u) \\
& +\frac{u}{1-u}+\frac{1-u}{4(1-2 u)}-u^{2}(1-u) S\left[U^{2}(x)\right] .
\end{aligned}
$$

Substituting the series assumption for $U(u)$ and Adomian polynomials for $U^{2}(x)$ and using (15) and (17), we obtain

$$
\begin{aligned}
& S\left(U_{0}(x)\right) \\
& =1-\frac{(1-u)}{4}-\frac{1}{2} u(1-u)+\frac{u}{1-u}+\frac{1-u}{4(1-2 u)} \\
& \quad S\left(U_{k+1}(x)\right)=-u^{2}(1-u) S\left[A_{k}(x)\right] .
\end{aligned}
$$

Taking the inverse Sumudu transform of both sides of (35) and using the recursive relation equation (36) give

$$
\begin{aligned}
& U_{0}(x)=1+x+x^{2}+\frac{1}{3} x^{3}+\frac{1}{8} x^{4}+\frac{1}{24} x^{5}+\cdots, \\
& U_{1}(x)=-\frac{1}{2} x^{2}-\frac{1}{6} x^{3}-\frac{1}{6} x^{4}-\frac{1}{12} x^{5}-\cdots, \\
& U_{2}(x)=\frac{1}{12} x^{4}+\frac{1}{20} x^{5}+\cdots .
\end{aligned}
$$

The series solution is given by

$$
U(x)=1+x+\frac{1}{2 !} x^{2}+\frac{1}{3 !} x^{3}+\frac{1}{4 !} x^{4}+\cdots,
$$

and the exact solution is

$$
U(x)=e^{x}
$$

\section{Systems of Nonlinear Volterra Integrodifferential Equations}

In this section, we will study systems of nonlinear Volterra integrodifferential equations of the second kind by combined Sumudu transform-Adomian decomposition method.

Consider systems of nonlinear Volterra integrodifferential equations of the second kind as follows:

$$
\begin{aligned}
U^{(n)}= & f_{1}(x) \\
& +\int_{0}^{x}\left(K_{1}(x-t) F_{1}(u(t))+R_{1}(x-t) G_{1}(v(t))\right) d t, \\
V^{(n)}= & f_{2}(x) \\
& +\int_{0}^{x}\left(K_{2}(x-t) F_{2}(u(t))+R_{2}(x-t) G_{2}(v(t))\right) d t .
\end{aligned}
$$

Applying Sumudu transforms to both sides of (40), we have

$$
\begin{aligned}
\frac{1}{u^{n}} S[U(x)]-\frac{1}{u^{n}} U(0)-\frac{1}{u^{n-1}} U^{\prime}(0)-\cdots-\frac{U^{n-1}(0)}{u} \\
=S\left(f_{1}(x)\right) \\
\quad+S\left(\left(K_{1}(x) * F_{1}(u(x))+R_{1}(x) * G_{1}(v(x))\right)\right), \\
\frac{1}{u^{n}} S[V(x)]-\frac{1}{u^{n}} V(0)-\frac{1}{u^{n-1}} V^{\prime}(0)-\cdots-\frac{V^{n-1}(0)}{u} \\
=S\left(f_{2}(x)\right) \\
\quad+S\left(\left(K_{2}(x) * F_{2}(u(x))+R_{2}(x) * G_{2}(v(x))\right)\right) .
\end{aligned}
$$

After rearrangement, we get

$$
\begin{aligned}
S[U(x)] & \\
= & U(0)+u U^{\prime}(0)+\cdots+u^{n-1} U^{(n-1)}(0)+u^{n} S\left(f_{1}(x)\right) \\
& +u^{n} S\left(\left(K_{1}(x) * F_{1}(u(x))+R_{1}(x) * G_{1}(v(x))\right)\right),
\end{aligned}
$$

$$
\begin{aligned}
& S[V(x)] \\
&= V(0)+u V^{\prime}(0)+\cdots+u^{n-1} V^{(n-1)}(0) \\
&+u^{n} S\left(f_{2}(x)\right) \\
&+u^{n} S\left(\left(K_{2}(x) * F_{2}(u(x))+R_{2}(x) * G_{2}(v(x))\right)\right) .
\end{aligned}
$$

To overcome the difficulty of the nonlinear terms $F_{i}(u(x)), i=1,2$, we apply Adomian decomposition method for handling (42) and (43). To achieve this goal, we first represent the linear terms $u(x)$ and $v(x)$ at the left side by an infinite series of components given by

$$
U(x)=\sum_{i=0}^{\infty} U_{i}(x), \quad V(x)=\sum_{i=0}^{\infty} V_{i}(x)
$$


and the nonlinear terms $F_{i}(u(x))$ at the right side of $(42)$ and (43) by

$$
\begin{aligned}
& F(u(t))=\sum_{n=0}^{\infty} A_{n}, \\
& A_{n}=\frac{1}{n !} \frac{d^{n}}{d \lambda^{n}}\left[F\left(\sum_{i=0}^{n} \lambda^{i} u_{i}\right)\right]_{\lambda=0}, \\
& n=0,1,2, \ldots,
\end{aligned}
$$

where Adomian polynomials $A_{n}, n \geq 0$, can be obtained for all forms of nonlinearity. Substituting (44) and (45) into (42) and (43) leads to

$$
\begin{aligned}
& S\left[\sum_{n=0}^{\infty} U_{n}(x)\right] \\
& =U(0)+u U^{\prime}(0)+\cdots+u^{n-1} U^{(n-1)}(0) \\
& \quad+u^{n} S\left(f_{1}(x)\right)+u^{n} S\left(K_{1}(x)\right) S\left(\left[\sum_{n=0}^{\infty} A_{n}\right]\right) \\
& \quad+u^{n} S\left(R_{1}(x)\right) S\left(\left[\sum_{n=0}^{\infty} \widetilde{A}_{n}\right]\right), \\
& S\left[\sum_{n=0}^{\infty} V_{n}(x)\right] \\
& =V(0)+u V^{\prime}(0)+\cdots+u^{n-1} V^{(n-1)}(0) \\
& \quad+u^{n} S\left(f_{2}(x)\right)+u^{n} S\left(K_{2}(x)\right) S\left(\left[\sum_{n=0}^{\infty} B_{n}\right]\right) \\
& \quad+u^{n} S\left(R_{2}(x)\right) S\left(\left[\sum_{n=0}^{\infty} B_{n}\right]\right) .
\end{aligned}
$$

Adomian decomposition method admits the use of the following recursive relations:

$$
\begin{aligned}
& S\left[U_{0}(x)\right]=U(0)+u U^{\prime}(0)+\cdots+u^{n-1} U^{(n-1)}(0) \\
& +u^{n} S\left(f_{1}(x)\right) \\
& S\left[U_{k+1}(x)\right] \\
& =u^{n} S\left(K_{1}(x)\right) S\left(A_{k}\right)+u^{n} S\left(R_{1}(x)\right) S\left(\widetilde{A_{k}}\right), \\
& S\left[V_{0}(x)\right]=V(0)+u V^{\prime}(0)+\cdots+u^{n-1} V^{(n-1)}(0) \\
& +u^{n} S\left(f_{2}(x)\right) \\
& S\left[V_{k+1}(x)\right] \\
& =u^{n} S\left(K_{2}(x)\right) S\left(B_{k}\right)+u^{n} S\left(R_{1}(x)\right) S\left(B_{k}\right) .
\end{aligned}
$$

The combined Sumudu transform-Adomian decomposition method for solving systems of nonlinear Volterra integrodifferential equations of the second kind will be illustrated by studying the following example.
Example 4. Solve the system of nonlinear Volterra integrodifferential equation by using the combined Sumudu transformAdomian decomposition method:

$$
\begin{aligned}
& \begin{aligned}
U^{\prime \prime}(x)= & \frac{7}{3} e^{x}-e^{2 x}-\frac{1}{3} e^{4 x} \\
& +\int_{0}^{x} e^{x-t}\left(U^{2}(t)+V^{2}(t)\right) d t, \\
V^{\prime \prime}(x)= & \frac{2}{3} e^{x}+3 e^{2 x}+\frac{1}{3} e^{4 x} \\
& +\int_{0}^{x} e^{x-t}\left(U^{2}(t)-V^{2}(t)\right) d t, \\
U(0)=1, & U^{\prime}(0)=1,
\end{aligned} \\
& V^{\prime}(0)=2 .
\end{aligned}
$$
obtain

Taking Sumudu transforms of both sides of (49), we

$$
\begin{aligned}
U(u)= & 1+u+\frac{7 u^{2}}{3(1-u)}-\frac{u^{2}}{(1-2 u)}-\frac{u^{2}}{3(1-4 u)} \\
& +\left(\frac{u^{3}}{1-u}\right) S\left[U^{2}(x)+V^{2}(x)\right], \\
V(u)= & 1+2 u+\frac{2 u^{2}}{3(1-u)}+\frac{3 u^{2}}{(1-2 u)}+\frac{u^{2}}{3(1-4 u)} \\
& +\left(\frac{u^{3}}{1-u}\right) S\left[U^{2}(x)-V^{2}(x)\right] .
\end{aligned}
$$

By using (48), we have

$$
\begin{aligned}
& U_{0}(u)=1+u+\frac{7 u^{2}}{3(1-u)}-\frac{u^{2}}{(1-2 u)}-\frac{u^{2}}{3(1-4 u)}, \\
& U_{k+1}=\left(\frac{u^{3}}{1-u}\right) S\left[A_{k}(x)+B_{k}(x)\right], \\
& V_{0}(u)=1+2 u+\frac{2 u^{2}}{3(1-u)}+\frac{3 u^{2}}{(1-2 u)}+\frac{u^{2}}{3(1-4 u)}, \\
& V_{k+1}=\left(\frac{u^{3}}{1-u}\right) S\left[A_{k}(x)-B_{k}(x)\right] .
\end{aligned}
$$

Recall that Adomian polynomials for $U^{2}(x)$ and $V^{2}(x)$ are given by

$$
\begin{aligned}
& A_{0}(x)=U_{0}^{2}, \quad A_{1}(x)=2 U_{0} U_{1}, \\
& A_{2}=2 U_{0} U_{2}+U_{1}^{2}, \\
& A_{3}(x)=2 U_{0} U_{3}+2 U_{1} U_{2}, \\
& B_{0}(x)=V_{0}^{2}, \quad B_{1}(x)=2 V_{0} V_{1}, \\
& B_{2}=2 V_{0} V_{2}+V_{1}^{2}, \quad B_{3}(x)=2 V_{0} V_{3}+2 V_{1} V_{2} .
\end{aligned}
$$


Taking the inverse Sumudu transform of both sides of (48) and using the recursive relation equations (48), we obtain the solution as follows:

$$
\begin{aligned}
& U(x)=\left(1+x+\frac{1}{2 !} x^{2}+\frac{1}{3 !} x^{3}+\frac{1}{4 !} x^{4}+\ldots\right) \\
& V(x)=\left(1+2 x+\frac{1}{2 !}(2 x)^{2}+\frac{1}{3 !}(2 x)^{3}+\frac{1}{4 !}(2 x)^{4}+\ldots\right)
\end{aligned}
$$

Then the solution for the above system is given by

$$
U(x)=e^{x}, \quad V(x)=e^{2 x}
$$

\section{Conclusion}

Sumudu transform-Adomian decomposition method has been applied to a system of nonlinear Volterra integrodifferential equations. Three examples have been presented; the method is very useful and reliable for any type of Volterra integrodifferential equations systems. Therefore, this method can even be applied to many complicated linear and nonlinear Volterra integrodifferential equations.

\section{Conflict of Interests}

The authors declare that there is no conflict of interests regarding the publication of this paper.

\section{Acknowledgment}

The authors would like to extend their sincere appreciation to the Deanship of Scientific Research at King Saud University for its funding of this research through the Research Group Project no. RGP-VPP-117.

\section{References}

[1] A.-M. Wazwaz, Linear and Nonlinear Integral Equations Methods and Applications, Springer, Higher Education Press, 2011.

[2] E. Alizadeh, M. Farhadi, K. Sedighi, H. R. Ebrahimi-Kebria, and A. Ghafourian, "Solution of the Falkner-Skan equation for wedge by Adomian Decomposition Method," Communications in Nonlinear Science and Numerical Simulation, vol. 14, no. 3, pp. 724-733, 2009.

[3] A. M. Wazwaz, "The modified decomposition method and Pade approximants for a boundary layer equation in unbounded domain," Applied Mathematics and Computation, vol. 177, no. 2, pp. 737-744, 2006.

[4] N. Bellomo and R. Monaco, "A Comparison between Adomian decomposition method and pertubation techniques for nonlinear random differential equations," Journal of Mathematical Analysis and Applications, vol. 110, no. 2, pp. 495-502, 1985.

[5] R. Race, "On the Adomian decomposition method and comparison with Picard's method," Journal of Mathematical Analysis and Applications, vol. 128, no. 2, pp. 480-483, 1987.

[6] G. Adomian and R. Race, "Noise terms in decomposition solution series," Computers \& Mathematics with Applications, vol. 24, no. 11, pp. 61-64, 1992.
[7] A. M. Wazwaz, "Necessary conditions for the appearance of noise terms in decomposition solution series," Applied Mathematics and Computation, vol. 81, no. 2-3, pp. 265-274, 1997.

[8] A. Kadem, Solving the One-Dimensional Neutron Transport Equation Using Chebyshev Polynomials and the Sumudu Transform, Analele Universitatii Din Oradea, Fascicola Matematica, 2005.

[9] H. Eltayeb, A. K. Kılıçman, and B. Fisher, "A new integral transform and associated distributions," Integral Transforms and Special Functions, vol. 21, no. 5, pp. 367-379, 2010.

[10] A. Kiliçman and H. E. Gadain, "On the applications of Laplace and Sumudu transforms," Journal of the Franklin Institute, vol. 347, no. 5, pp. 848-862, 2010.

[11] A. Kiliçman, H. Eltayeb, and P. R. Agarwal, "On sumudu transform and system of differential equations," Abstract and Applied Analysis, vol. 2010, Article ID 598702, 11 pages, 2010.

[12] A. Kiliçman, H. Eltayeb, and K. A. M. Atan, "A note on the comparison between laplace and sumudu transforms," Bulletin of the Iranian Mathematical Society, vol. 37, no. 1, pp. 131-141, 2011.

[13] F. B. M. Belgacem, A. A. Karaballi, and S. L. Kalla, "Analytical investigations of the sumudu transform and applications to integral production equations," Mathematical Problems in Engineering, vol. 2003, no. 3-4, pp. 103-118, 2003. 


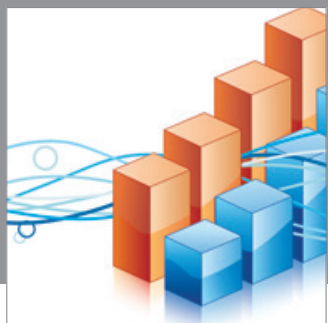

Advances in

Operations Research

mansans

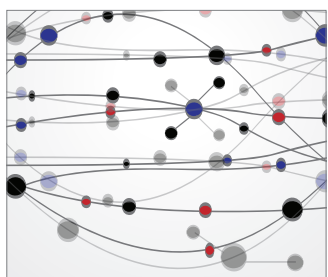

The Scientific World Journal
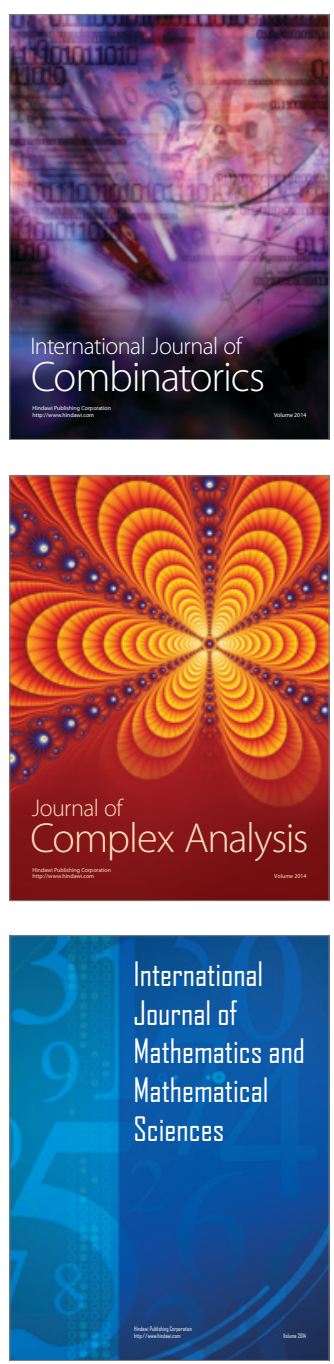
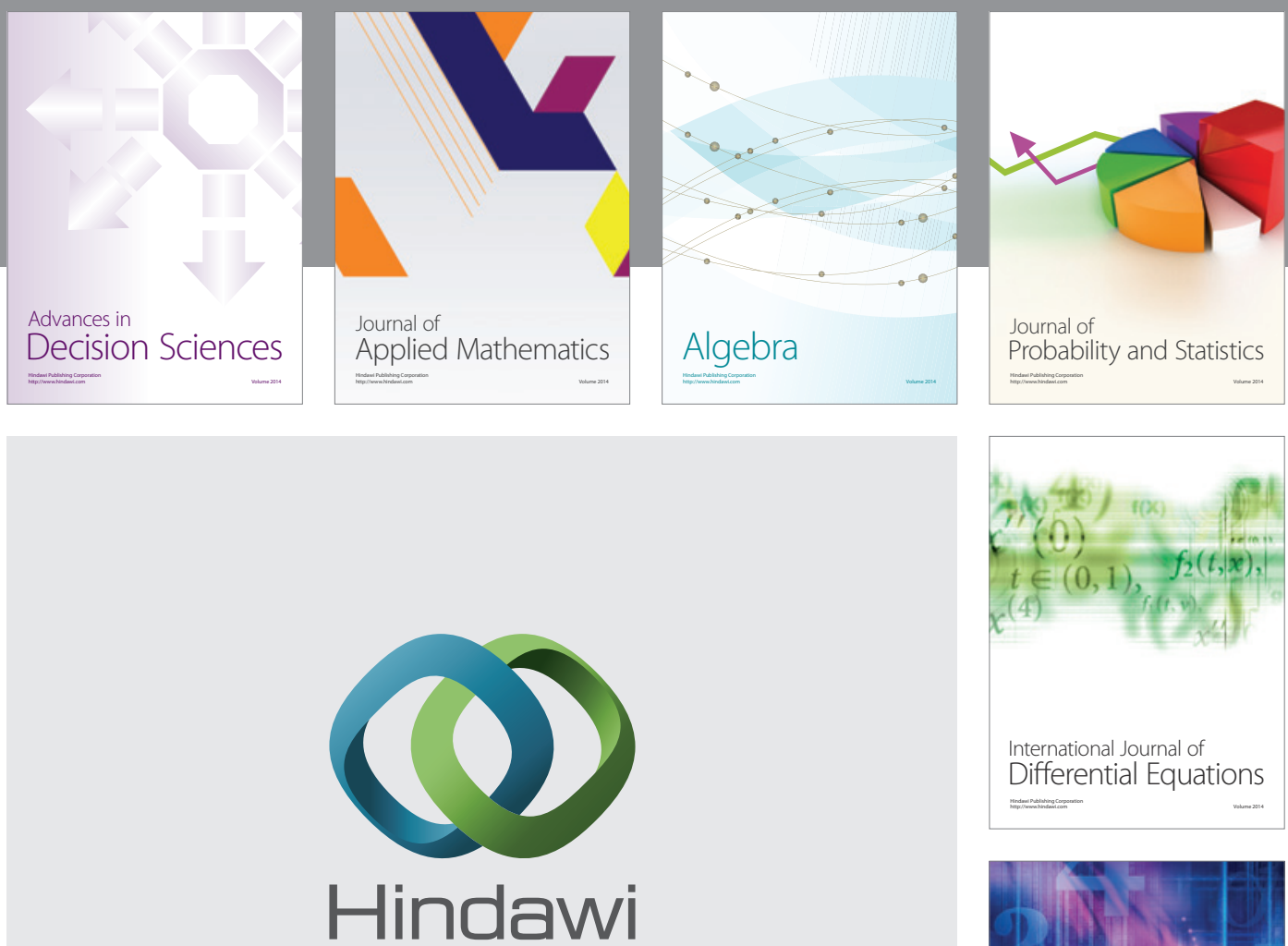

Submit your manuscripts at http://www.hindawi.com
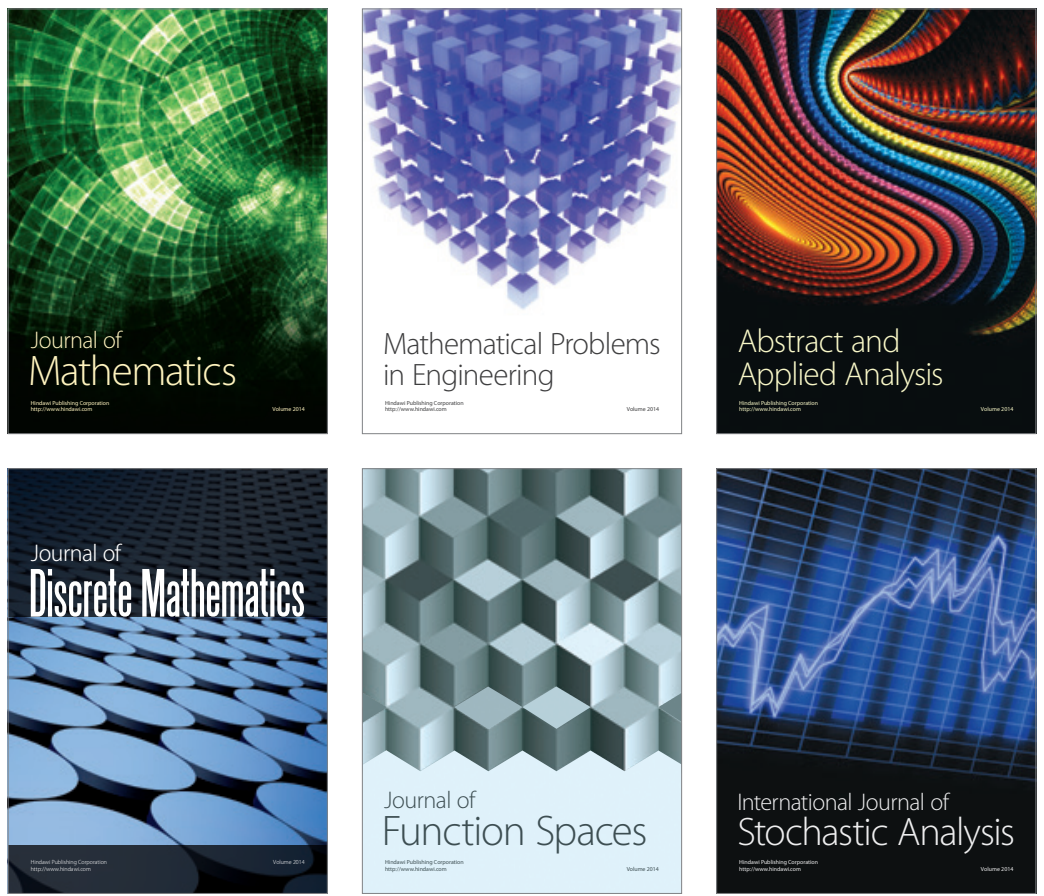

Journal of

Function Spaces

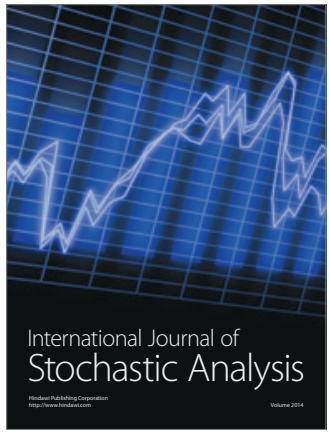

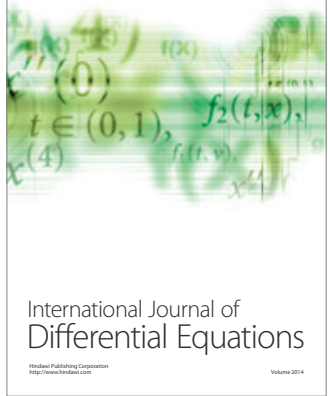
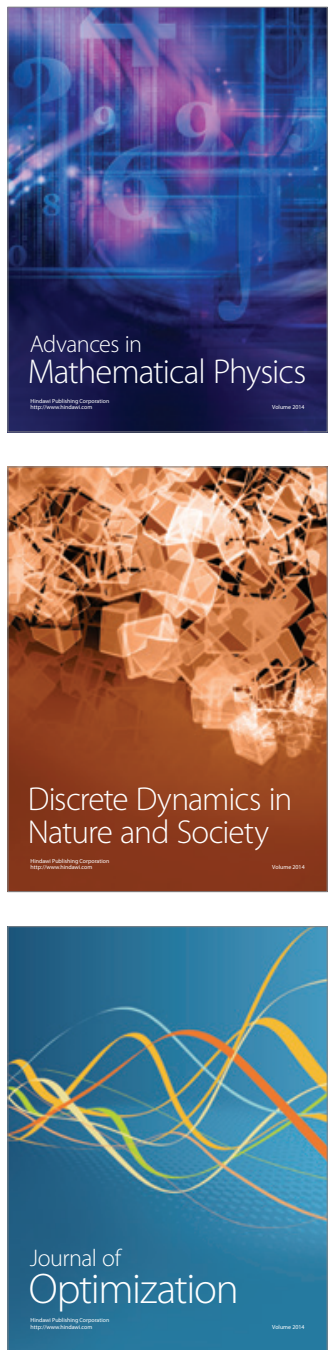